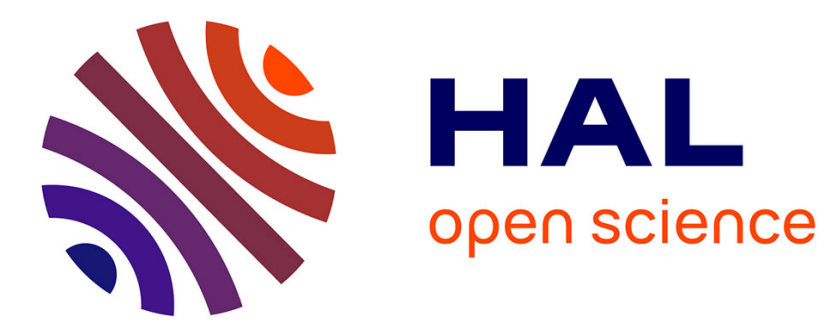

\title{
Order, type and cotype of growth for p-adic entire functions A survey with additional properties
}

Kamal Boussaf, Abdelbaki Boutabaa, Alain Escassut

\section{To cite this version:}

Kamal Boussaf, Abdelbaki Boutabaa, Alain Escassut. Order, type and cotype of growth for p-adic entire functions A survey with additional properties. p-Adic Numbers, Ultrametric Analysis and Applications, 2016. hal-01918234

\section{HAL Id: hal-01918234 \\ https://hal.uca.fr/hal-01918234}

Submitted on 10 Nov 2018

HAL is a multi-disciplinary open access archive for the deposit and dissemination of scientific research documents, whether they are published or not. The documents may come from teaching and research institutions in France or abroad, or from public or private research centers.
L'archive ouverte pluridisciplinaire $\mathbf{H A L}$, est destinée au dépôt et à la diffusion de documents scientifiques de niveau recherche, publiés ou non, émanant des établissements d'enseignement et de recherche français ou étrangers, des laboratoires publics ou privés. 


\title{
Order, type and cotype of growth for $p$-adic entire functions
}

\section{A survey with additional properties}

\author{
Kamal Boussaf, Abdelbaki Boutabaa
}

\section{and Alain Escassut}

\begin{abstract}
Let $\mathbb{I K}$ be a complete ultrametric algebraically closed field and let $\mathcal{A}(\mathbb{K})$ be the $\mathbb{I K}$-algebra of entires functions on $\mathbb{K}$. For an $f \in \mathcal{A}(\mathbb{K})$, similarly to complex analysis, one can define the order of growth as $\rho(f)=\limsup _{r \rightarrow+\infty} \frac{\log (\log (|f|(r))}{\log r}$. When $\rho(f) \neq 0,+\infty$, one can define the type of growth as $\sigma(f)=\limsup _{r \rightarrow+\infty} \frac{\log (|f|(r))}{r^{\rho(f)}}$. But here, we can also define the cotype of growth as $\psi(f)=\limsup _{r \rightarrow+\infty} \frac{q(f, r)}{r^{\rho(f)}}$ where $q(f, r)$ is the number of zeros of $f$ in the disk of center 0 and radius $r$. Many properties described here were first given in the Houston Journal, but new inequalities linking the order, type and cotype are given in this paper: we show that $\rho(f) \sigma(f) \leq \psi(f) \leq e \rho(f) \sigma(f)$. Moreover, if $\psi$ or $\sigma$ are veritable limits, then $\rho(f) \sigma(f)=\psi(f)$ and this relation is conjectured in the general case. Several other properties are examined concerning $\rho, \sigma, \psi$ for $f$ and $f^{\prime}$. Particularly, we show that if an entire function $f$ has finite order, then $\frac{f^{\prime}}{f^{2}}$ takes every value infinitely many times.
\end{abstract}

Keywords: p-adic entire functions, growth of entire functions, order, type and cotype of growth.

2010 Mathematics Subject Classification: 12J25; 30D35; 30G06; 46 S10.

\section{Order and cotype of growth}

We denote by $\mathbb{K}$ an algebraically closed field of characteristic 0 , complete with respect to an ultrametric absolute value $\mid$. |. Analytic functions inside a disk or in the whole field $\mathbb{K}$ were introduced and studied in many books. Given $\alpha \in \mathbb{K}$ and $R \in \mathbb{R}_{+}^{*}$, we denote by $d(\alpha, R)$ the disk $\{x \in \mathbb{K}|| x-\alpha \mid \leq R\}$, by $d\left(\alpha, R^{-}\right)$the disk $\{x \in \mathbb{K}|| x-\alpha \mid<R\}$, by $C(\alpha, r)$ the circle $\{x \in \mathbb{K}|| x-\alpha \mid=r\}$, by $\mathcal{A}(\mathbb{K})$ the $\mathbb{K}$-algebra of analytic functions in $\mathbb{K}$ (i.e. the set of power series with an infinite radius of convergence) and by $\mathcal{M}(\mathbb{K})$ the field of meromorphic functions in $\mathbb{K}$ (i.e. the field of fractions of $\mathcal{A}(\mathbb{K})$ ). Given $f \in \mathcal{M}(\mathbb{K})$, we will denote by $q(f, r)$ the number of zeros of $f$ in $d(0, r)$, taking multiplicity into account and by $u(f, r)$ the number of distinct multiple poles of $f$ in $d(0, r)$. Throughout the paper, log denotes the Neperian logarithm.

Here we mean to introduce and study the notion of order of growth and type of growth for functions of order $t$. We will also introduce a new notion of cotype of growth in relation with the distribution of zeros in disks which plays a major role in processes that are quite 
different from those in complex analysis. This has an application to the question whether an entire function can be devided by its derivative inside the algebra of entire functions.

Let us shortly recall classical results [5], [6], [8]:

Notation: Given $f \in \mathcal{A}(\mathbb{K})$ and $r>0$, we denote by $|f|(r)$ the number $\sup \{|f(x)||| x \mid=r\}$.

Theorem A $||.(r)$ is a multiplicative norm on $\mathcal{A}(\mathbb{K})$. Suppose $f(0) \neq 0$ and let $a_{1}, \ldots, a_{m}$ be the various zeros of $f$ in $d(0, r)$ with $\left|a_{n}\right| \leq\left|a_{n+1}\right|, 1 \leq n \leq m-1$, each zero $a_{n}$ having a multiplicity order $w_{n}$. Then

$$
\log (|f|(r))=\log (|f(0)|)+\sum_{n=1}^{m} w_{n}\left(\log (r)-\log \left(\left|a_{n}\right|\right)\right) .
$$

Theorem B: Let $f \in \mathcal{A}(\mathbb{K})$ be non-identically zero and let $\left.r^{\prime}, r^{\prime \prime} \in\right] 0,+\infty\left[\right.$ with $r^{\prime}<r^{\prime \prime}$. Then

$$
\left(\frac{r^{\prime \prime}}{r^{\prime}}\right)^{q\left(f, r^{\prime \prime}\right)} \geq \frac{|f|\left(r^{\prime \prime}\right)}{|f|\left(r^{\prime}\right)} \geq\left(\frac{r^{\prime \prime}}{r^{\prime}}\right)^{q\left(f, r^{\prime}\right)} .
$$

Theorem C: Let $f \in \mathcal{A}(\mathbb{K})$. Then

$$
\left|f^{\prime}\right|(r) \leq \frac{|f|(r)}{r} \forall r>0
$$

Theorem D: Let $f, g \in \mathcal{A}(\mathbb{K})$. Then $|f \circ g|(r)=|f|(|g|(r)) \forall r>0$.

Here, we must recall a theorem proven in [3], (Main Theorem) and in [7] (Theorem 33.12), to characterize meromorphic functions admitting a primitive:

Theorem E: Let $f \in \mathcal{M}(\mathbb{K})$. Then $f$ admits primitives if and only if all its residues are null.

The question whether the derivative of a meromorphic function in $\mathbb{K}$ can admit a Picard value has been thoroughly examined. By [1] we have the following theorem:

Theorem F: Let $f \in \mathcal{M}(\mathbb{K})$ be such that there exists $c$ and $s \in] 0,+\infty[$ satisfying $u(f, r) \leq c r^{s} \forall r \geq 1$. Then for every $b \in \mathbb{K}, f^{\prime}-b$ has infinitely many zeros.

Definition and notation: Similarly to the definition known on complex entire functions $[9]$, given $f \in \mathcal{A}(\mathbb{K})$, the superior limit

$$
\limsup _{r \rightarrow+\infty}\left(\frac{\log (\log (|f|(r)))}{\log (r)}\right)
$$


is called the order of growth of $f$ or the order of $f$ in brief and is denoted by $\rho(f)$. We say that $f$ has finite order if $\rho(f)<+\infty$.

Theorem 1: Let $f, g \in \mathcal{A}(\mathbb{K})$. Then:

if $c(|f|(r))^{\alpha} \geq|g|(r)$ with $\alpha$ and $c>0$, when $r$ is big enough, then $\rho(f) \geq \rho(g)$, $\rho(f+g) \leq \max (\rho(f), \rho(g))$ $\rho(f g)=\max (\rho(f), \rho(g))$.

Proof: Similarly to the complex context we can easily verify that

$$
\rho(f+g) \leq \max (\rho(f), \rho(g)), \rho(f g) \leq \max (\rho(f), \rho(g))
$$

and if $c(|f|(r))^{\alpha} \geq|g|(r)$ with $\alpha$ and $c>0$ when $r$ is big enough, then $\rho(f) \geq \rho(g)$. Let us now show that $\rho(f g) \geq \max (\rho(f), \rho(g))$. Since $\lim _{r \rightarrow+\infty}|g|(r)=+\infty$, of course we have $\log (|f . g|(r)) \geq \log (|f|(r))$ when $r$ is big enough, hence

$$
\frac{\log (\log (|f \cdot g|(r)))}{\log (r)} \geq \frac{\log (\log (|f|(r)))}{\log (r)}
$$

and therefore $\rho(f . g) \geq \rho(f)$ and similarly, $\rho(f . g) \geq \rho(g)$.

Corollary 1.1: Let $f, g \in \mathcal{A}(\mathbb{K})$. Then $\rho\left(f^{n}\right)=\rho(f) \forall n \in \mathbb{N}^{*}$. If $\rho(f)>\rho(g)$, then $\rho(f+g)=\rho(f)$.

Remark: $\rho$ is an ultrametric extended semi-norm.

Notation: Given $t \in[0,+\infty[$, we denote by $\mathcal{A}(\mathbb{K}, t)$ the set of $f \in \mathcal{A}(\mathbb{K})$ such that $\rho(f) \leq t$ and we set

$$
\mathcal{A}^{0}(\mathbb{K})=\bigcup_{t \in[0,+\infty[} \mathcal{A}(\mathbb{K}, t)
$$

Corollary 1.2. For any $t \geq 0, \mathcal{A}(\mathbb{K}, t)$ is a $\mathbb{K}$-subalgebra of $\mathcal{A}(\mathbb{K})$. If $s \leq t$, then $\mathcal{A}(\mathbb{K}, s) \subset \mathcal{A}(\mathbb{K}, t)$ and $\mathcal{A}^{0}(\mathbb{K})$ is also a $\mathbb{K}$-subalgebra of $\mathcal{A}(\mathbb{K})$.

Theorem 2: Let $f \in \mathcal{A}(\mathbb{K})$ and let $P \in \mathbb{K}[x]$. Then $\rho(P \circ f)=\rho(f)$ and $\rho(f \circ P)=\operatorname{deg}(P) \rho(f)$.

Proof: Let $n=\operatorname{deg}(P)$. For $r$ big enough, we have

$\log (\log (|f|(r))) \leq \log (\log (|P \circ f|(r))) \leq \log ((n+1) \log (|f|(r)))=\log (n+1)+\log (\log (|f|(r)))$

Consequently,

$$
\limsup _{r \rightarrow+\infty}\left(\frac{\log (\log (|f|(r)))}{\log (r)}\right) \leq \limsup _{r \rightarrow+\infty}\left(\frac{\log (\log (|P \circ f|(r)))}{\log (r)}\right)
$$




$$
\leq \limsup _{r \rightarrow+\infty}\left(\frac{\log (n+1)+\log (\log (|f|(r)))}{\log (r)}\right)
$$

and therefore $\rho(P \circ f)=\rho(f)$.

Next, for $r$ big enough, we have

$$
\frac{\log (\log (|f|(r)))}{\log (r)} \leq \frac{\log (\log (|f \circ P|(r)))}{\log (r)}=\left(\frac{\log (\log (|f \circ P|(r)))}{\log (|P|(r))}\right)\left(\frac{\log (|P|(r))}{\log (r)}\right)
$$

Now,

$$
\limsup _{r \rightarrow+\infty}\left(\frac{\log (\log (|f \circ P|(r)))}{\log (|P|(r))}\right)=\limsup _{r \rightarrow+\infty}\left(\frac{\log (\log (|f|(r)))}{\log (r)}\right)
$$

because the function $h$ defined in $[0,+\infty[$ as $h(r)=|P|(r)$ is obviously an increasing continuous bijection from $[0,+\infty[$ onto $[|P(0)|,+\infty[$. On the other hand, it is obviously seen that $\limsup _{r \rightarrow+\infty}\left(\frac{\log (|P|(r))}{\log (r)}\right)=n$. Consequently,

$$
\limsup _{r \rightarrow+\infty}\left(\frac{\log (\log (|f \circ P|(r)))}{\log (|P|(r))}\right)=n \limsup _{r \rightarrow+\infty}\left(\frac{\log (\log (|f|(r)))}{\log (r)}\right)
$$

and hence $\rho(f \circ P)=n \rho(f)$.

Theorem 3: Let $f, g \in \mathcal{A}(\mathbb{K})$ be transcendental. If $\rho(f) \neq 0$, then $\rho(f \circ g)=+\infty$. If $\rho(f)=0$, then $\rho(f \circ g) \geq \rho(g)$.

Proof: Let us fix an integer $n \in \mathbb{N}$. Let $f(x)=\sum_{j=0}^{\infty} a_{n} x^{n}$ and $g(x)=\sum_{j=0}^{\infty} b_{n} x^{n}$. Since $g$ is transcendental, for every $n \in \mathbb{N}$, there exists $r_{n}$ such that $q\left(g, r_{n}\right) \geq n$. Then $|g|(r) \geq\left|b_{n}\right| r^{n} \forall r \geq r_{n}$ and hence, by Theorem 2, we have

$$
\rho(f \circ g) \geq n \rho(f) .
$$

Relation (1) is true for every $n \in \mathbb{N}$. Suppose first that $\rho(f) \neq 0$. Then by (1) we have $\rho(f \circ g)=+\infty$.

Now, suppose $\rho(f)=0$. Let $k \in \mathbb{N}$ be such that $a_{k} \neq 0$. Let $s_{0}$ be such that $q\left(f, s_{0}\right) \geq k$. Then $|f|(r) \geq\left|a_{k}\right| r^{k} \forall r \geq s_{0}$, hence $|f \circ g|(r) \geq\left|a_{k}\right|(|g|(r))^{k} \forall r \geq s_{0}$, hence by Theorems 1 and 2 we have $\rho(f \circ g) \geq \rho(g)$.

Theorem 4: Let $f \in \mathcal{A}(\mathbb{K})$ be not identically zero. If there exists $s \geq 0$ such that

$$
\limsup _{r \rightarrow+\infty}\left(\frac{q(f, r)}{r^{s}}\right)<+\infty
$$

then $\rho(f)$ is the lowest bound of the set of $s \in[0,+\infty[$ such that

$$
\limsup _{r \rightarrow+\infty}\left(\frac{q(f, r)}{r^{s}}\right)=0 \text {. }
$$


Moreover, if $\limsup _{r \rightarrow+\infty}\left(\frac{q(f, r)}{r^{t}}\right)$ is a number $\left.b \in\right] 0,+\infty[$, then $\rho(f)=t$.

If there exists no s such that $\limsup _{r \rightarrow+\infty}\left(\frac{q(f, r)}{r^{s}}\right)<+\infty$, then $\rho(f)=+\infty$.

Proof: The proof holds in two statements. First we will prove that given $f \in \mathcal{A}(\mathbb{K})$ nonconstant and such that for some $t \geq 0, \limsup _{r \rightarrow+\infty} \frac{q(f, r)}{r^{t}}$ is finite, then $\rho(f) \leq t$.

Set $\limsup _{r \rightarrow+\infty}\left(\frac{q(f, r)}{r^{t}}\right)=b \in[0,+\infty[$. Let us fix $\epsilon>0$. We can find $R>1$ such that $|f|(R)>e^{2}$ and $\frac{q(f, r)}{r^{t}} \leq b+\epsilon \forall r \geq R$ and hence, by Theorem B, we have $\frac{|f|(r)}{|f|(R)} \leq$ $\left(\frac{r}{R}\right)^{q(f, r)} \leq\left(\frac{r}{R}\right)^{\left.r^{t}(b+\epsilon)\right)}$. Therefore, since $R>1$, we have

$$
\log (|f|(r)) \leq \log (|f|(R))+r^{t}(b+\epsilon)(\log (r))
$$

Now, when $u>2, v>2$, we check that $\log (u+v) \leq \log (u)+\log (v)$. Applying that inequality with $u=\log (|f|(R))$ and $v=r^{t}(b+\epsilon)(\log (r))$ when $r^{t}(b+\epsilon)(\log (r))>2$, that yields

$$
\log (\log (|f|(r))) \leq \log (\log (|f|(R)))+t \log (r)+\log (b+\epsilon)+\log (\log (r)) .
$$

Consequently,

$$
\frac{\log (\log (|f|(r)))}{\log (r)} \leq \frac{\log (\log (|f|(R)))+t \log (r)+\log (b+\epsilon)+\log (\log (r))}{\log (r)}
$$

and hence we can check that

$$
\limsup _{r \rightarrow+\infty} \frac{\log (\log (|f|(r)))}{\log (r)} \leq t
$$

which proves the first claim.

Second, we will prove that given $f \in \mathcal{A}(\mathbb{K})$ not identically zero and such that for some $t \geq 0$, we have $\limsup _{r \rightarrow+\infty} \frac{q(f, r)}{r^{t}}>0$, then $\rho(f) \geq t$.

By hypotheses, there exists a sequence $\left(r_{n}\right)_{n \in \mathbb{N}}$ such that $\lim _{n \rightarrow+\infty} r_{n}=+\infty$ and

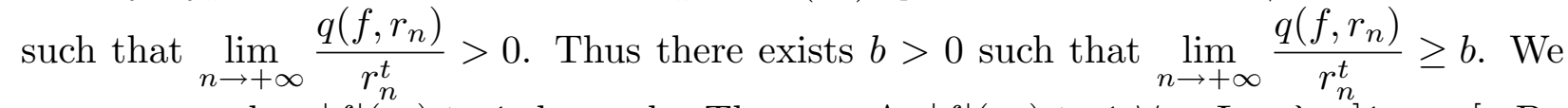
can assume that $|f|\left(r_{0}\right) \geq 1$, hence by Theorem A, $|f|\left(r_{n}\right) \geq 1 \forall n$. Let $\left.\lambda \stackrel{n}{\in}\right] 1,+\infty[$. By Theorem B we have

$$
\frac{|f|\left(\lambda r_{n}\right)}{|f|\left(r_{n}\right)} \geq(\lambda)^{q\left(f, r_{n}\right)} \geq(\lambda)^{\left[b\left(r_{n}\right)^{t}\right]}
$$

hence

$$
\log \left(|f|\left(\lambda r_{n}\right) \geq \log \left(|f|\left(r_{n}\right)\right)+b\left(r_{n}\right)^{t} \log (\lambda)\right.
$$


Since $|f|\left(r_{n}\right) \geq 1$, we have $\log \left(\log \left(|f|\left(\lambda r_{n}\right)\right)\right) \geq \log (b \log (\lambda))+t \log \left(r_{n}\right)$ therefore

$$
\frac{\log \left(\log \left(|f|\left(\lambda r_{n}\right)\right)\right.}{\log \left(r_{n}\right)} \geq t+\frac{\log (b \log (\lambda))}{\log \left(r_{n}\right)} \forall n \in \mathbb{N}
$$

and hence

$$
\limsup _{r \rightarrow+\infty} \frac{\log (\log (|f|(r)))}{\log (r)} \geq t
$$

which ends the proof the scond claim.

Example: Suppose that for each $r>0$, we have $q(f, r) \in\left[r^{t} \log r, r^{t} \log r+1\right]$. Then of course, for every $s>t$, we have $\limsup _{r \rightarrow+\infty} \frac{q(f, r)}{r^{s}}=0$ and $\limsup _{r \rightarrow+\infty} \frac{q(f, r)}{r^{t}}=+\infty$, so there exists no $t>0$ such that $\frac{q(f, r)}{r^{t}}$ have non-zero superior limit $b<+\infty$.

Definition and notation: Let $t \in[0,+\infty[$ and let $f \in \mathcal{A}(\mathbb{K})$ of order $t$. We set $\psi(f)=\limsup _{r \rightarrow+\infty} \frac{q(f, r)}{r^{t}}$ and call $\psi(f)$ the cotype of $f$.

Theorem 5: Let $f, g \in \mathcal{A}^{0}(\mathbb{K})$. Then $\psi(f g) \leq \psi(f)+\psi(g)$. Moreover, if $\rho(f)=\rho(g)$ then $\max (\psi(f), \psi(g)) \leq \psi(f g)$.

Proof: Set $\rho(f)=s, \rho(g)=t$. Without loss of generality we can assume $s \geq t$. By Theorem 1, we have $\rho(f . g)=\rho(f)=s$. Now, for each $r>0$, we have $q(f . g, r)=$ $q(f, r)+q(g, r)$ hence

$$
\psi(f g)=\limsup _{r \rightarrow+\infty} \frac{q(f, r)+q(g, r)}{r^{s}} \leq \limsup _{r \rightarrow+\infty} \frac{q(f, r)}{r^{s}}+\limsup _{r \rightarrow+\infty} \frac{q(g, r)}{r^{t}}
$$

hence $\psi(f g) \leq \psi(f)+\psi(g)$.

Now, suppose $s=t$. Then

$$
\psi(f g)=\limsup _{r \rightarrow+\infty} \frac{q(f, r)+q(g, r)}{r^{s}} \geq \limsup _{r \rightarrow+\infty} \frac{\max (q(f, r), q(g, r))}{r^{s}}=\max (\psi(f), \psi(g)),
$$

which ends the proof.

Thanks to Theorem $\mathrm{F}$ we can derive the following Theorem 6:

Theorem 6: Let $f \in \mathcal{A}^{0}(\mathbb{K})$. Then for every $b \in \mathbb{K}, \frac{f^{\prime}}{f^{2}}-b$ has infinitely many zeros. Proof Let $t=\rho(f)$ and take $c>\psi(f)$. Since $f \in \mathcal{A}^{0}(\mathbb{K})$, by Theorem 4 we have $q(f, r) \leq c r^{t}$ when $r$ is big enough, hence by Theorem $\mathrm{F}$ the derivative of $\frac{1}{f}$ takes every value $b$ infinitely many times. 
Now we can derive the following Corollary 6.1 that gives the solution for a function of finite order, of the following general problem: given an entire function $f$, is it posible that all zeros of $f^{\prime}$ be zeros of $f$, but finitely many?

Corollary 6.1: Let $f \in \mathcal{A}^{0}(\mathbb{K})$. Then $f^{\prime}$ admits infinitely many zeros that are not zeros of $f$.

Theorem 7 is similar to a well known statement in complex analysis and its proof also is similar when $\rho(f)<+\infty[9]$ but is different when $\rho(f)=+\infty$.

Theorem 7 Let $f(x)=\sum_{n=0}^{+\infty} a_{n} x^{n} \in \mathcal{A}(\mathbb{K})$. Then $\rho(f)=\limsup _{n \rightarrow+\infty}\left(\frac{n \log (n)}{-\log \left|a_{n}\right|}\right)$.

Proof: If $\rho(f)<+\infty$, the proof is identical to the one made in the complex context, replacing $M(f, r)$ by the multiplicative norm $|f|(r)$. Let $t=\rho(f)$ and suppose first that $t<+\infty$. Let $\alpha=\lim \sup _{n \rightarrow+\infty} \frac{n \log (n)}{-\log \left|a_{n}\right|}$. Take $s>t$. For all $n \in \mathbb{N}$, we have $\left|a_{n}\right| r^{n} \leq$ $|f|(r)$ and therefore $\left|a_{n}\right| r^{n} \leq e^{\left(r^{s}\right)}$ and hence $\left|a_{n}\right| \leq r^{-n} e^{\left(r^{s}\right)}$ i.e.

$$
\log \left|a_{n}\right| \leq r^{s}-n \log (r)
$$

when $r$ is big enough.

Now, choose $r=\left(\frac{n}{s}\right)^{\frac{1}{s}}$. So, we have $\log \left|a_{n}\right| \leq \frac{n}{s}-\frac{n}{s} \log \left(\frac{n}{s}\right)$, i.e.

$-\log \left(\left|a_{n}\right|\right) \geq-\frac{n}{s}+\frac{n}{s} \log \left(\frac{n}{s}\right)$.

Consequently, when $n$ is big enough we have

$$
\frac{n \log n}{\left(-\log \left|a_{n}\right|\right)} \leq \frac{n \log n}{\frac{n}{s} \log \left(\frac{n}{s}\right)-\frac{n}{s}} \leq s+O(1)
$$

Therefore we have $\alpha \leq s$ and since this is true for each $s>t$, that shows that $\alpha \leq t$.

Now, take $\beta>\alpha$ so that $\frac{n \log n}{\left(-\log \left|a_{n}\right|\right)}<\beta$ for $n$ big enough. Then, when $n$ is big enough, we have $n \log (n) \leq \beta\left(-\log \left|a_{n}\right|\right)$ hence $n^{\frac{n}{\beta}} \leq \frac{1}{\left|a_{n}\right|}$ and hence $\left|a_{n}\right| \leq \frac{1}{n^{\frac{n}{\beta}}}$. Consequently, $\left|a_{n}\right| r^{n} \leq \frac{r^{n}}{n^{\frac{n}{\beta}}}$. Now, for $r$ big enough, $|f|(r)=\sup _{n \in \mathbb{N}}\left|a_{n}\right| r^{n} \leq \sup _{n \in \mathbb{N}} \frac{r^{n}}{n^{\frac{n}{\beta}}}$.

Putting $\varphi(n)=\frac{n}{\beta}$ and $R=\frac{r}{\beta}$, we have

$$
|f|\left(r^{\frac{1}{\beta}}\right) \leq \sup _{n \in \mathbb{N}} \frac{r^{\varphi(n)}}{n^{\varphi(n)}} \leq \sup _{x>0} \frac{R^{x}}{x^{x}}
$$

Now we check that the maximum on $\left[0,+\infty\left[\right.\right.$ of the function $g(x)=\frac{R^{x}}{x^{x}}$ is reached when $x=\frac{R}{e}$ and hence is $e^{\frac{R}{e}}=e^{\frac{r}{\beta e}}$. Therefore, we have $|f|\left(r^{\frac{1}{\beta}}\right) \leq e^{\frac{r}{\beta e}}$. Putting now $u=r^{\frac{1}{\beta}}$, we can derive $|f|(u) \leq e^{\frac{u^{\beta}}{\beta e}}$, hence

$$
\log (\log (|f|(u))) \leq \beta \log (u)-\log (e \beta) .
$$


Consequently,

$$
\limsup _{r \rightarrow+\infty} \frac{\log (\log (|f|(r)))}{\log (r)} \leq \beta
$$

So we have $t \leq \beta$ and since this is true for all $\beta>\alpha$, we have proven that $t \leq \alpha$, which ends the proof when $t<+\infty$.

Suppose now that $t=+\infty$ and suppose that $\limsup _{n \rightarrow+\infty} \frac{n \log n}{\left(-\log \left|a_{n}\right|\right)}<+\infty$. Let us take $s \in \mathbb{N}$ such that

$$
\frac{n \log n}{\left(-\log \left|a_{n}\right|\right)}<s \forall n \in \mathbb{N}
$$

By Theorem 4, we have $\limsup _{r \rightarrow+\infty} \frac{q(f, r)}{s}=+\infty$. So, we can take a sequence $\left(r_{m}\right)_{m \in \mathbb{N}}$ such that

$$
\lim _{m \rightarrow+\infty} \frac{q\left(f, r_{m}\right)}{\left(r_{m}\right)^{s}}=+\infty
$$

For simplicity, set $u_{m}=q\left(f, r_{m}\right), m \in \mathbb{N}$. By $(2)$, for $m$ big enough we have

$$
u_{m} \log \left(u_{m}\right)<s\left(-\log \left(\left|a_{u_{m}}\right|\right)=s \log \left(\frac{1}{\left|a_{u_{m}}\right|}\right)\right.
$$

hence

$$
\frac{1}{\left(u_{m}\right)^{u_{m}}}>\left|a_{u_{m}}\right|^{s}
$$

therefore

$$
\left|a_{u_{m}}\right|^{s}\left(r_{m}\right)^{s u_{m}}<\frac{\left(r_{m}\right)^{s u_{m}}}{\left(u_{m}\right)^{u_{m}}}
$$

i.e.

$$
\left(|f|\left(r_{m}\right)\right)^{s}<\left(\frac{\left(r_{m}\right)^{s}}{u_{m}}\right)^{u_{m}}
$$

But by Theorem A, we have $\lim _{r \rightarrow+\infty}|f|\left(r_{m}\right)=+\infty$, hence $\left(r_{m}\right)^{s}>u_{m}$ when $m$ is big enough and therefore $\limsup _{m \rightarrow+\infty} \frac{q\left(f, r_{m}\right)}{\left(r_{m}\right)^{s}} \leq 1$, a contradiction to (3). Consequently, (2) is impossible and therefore

$$
\limsup _{n \rightarrow+\infty}\left(\frac{n \log (n)}{-\log \left|a_{n}\right|}\right)=+\infty=\rho(f) .
$$

Remark: Of course, polynomials have a growth order equal to 0 . On $\mathbb{I K}$ as on $\mathbb{C}$ we can easily construct transcendental entire functions of order 0 or of order $\infty$. 
Example 1: Let $\left(a_{n}\right)_{n \in \mathbb{N}}$ be a sequence in $\mathbb{K}$ such that $-\log \left|a_{n}\right| \in\left[n(\log n)^{2}, n(\log n)^{2}+1\right]$. Then clearly,

$$
\lim _{n \rightarrow+\infty} \frac{\log \left|a_{n}\right|}{n}=-\infty
$$

hence the function $\sum_{n=0}^{\infty} a_{n} x^{n}$ has a radius of convergence equal to $+\infty$. On the other hand,

$$
\lim _{n \rightarrow+\infty} \frac{n \log n}{-\log \left|a_{n}\right|}=0
$$

hence $\rho(f)=0$.

Example 2: $\quad$ Let $\left(a_{n}\right)_{n \in \mathbb{N}}$ be a sequence in $\mathbb{K}$ such that $-\log \left|a_{n}\right| \in[n \sqrt{\log n}, n \sqrt{\log n}+1]$. Then

$$
\lim _{n \rightarrow+\infty} \frac{\log \left|a_{n}\right|}{n}=-\infty
$$

again and hence the function $\sum_{n=0}^{\infty} a_{n} x^{n}$ has a radius of convergence equal to $+\infty$. On the other hand, $\lim _{n \rightarrow+\infty}\left(\frac{n \log n}{-\log \left|a_{n}\right|}\right)=+\infty$, hence $\rho(f)=+\infty$.

Thanks to Theorem F, we can now prove Theorem 8:

Theorem 8: Let $f=\frac{g}{h} \in \mathcal{M}(\mathbb{K})$ with $g \in \mathcal{A}(\mathbb{K})$ and $h \in \mathcal{A}^{0}(\mathbb{K})$ and $\psi(h)<+\infty$. Then for every $b \in \mathbb{K}, f^{\prime}-b$ has infinitely many zeros.

Proof: Set $t=\rho(h)$. There exists $\ell>\psi(h)$ such that $q(r, h) \leq \ell r^{t} \forall r>1$. Consequently, taking $s>t$ big enough, we have $u(f, r)<r^{s} \forall r>1$ and hence $f$ satisfies the hypotheses of Theorem F. Therefore, for every $b \in \mathbb{K}, f^{\prime}-b$ has infinitely may zeros.

Then by Theorem E, we can now derive Corollary 8.1:

Corollary 8.1: Let $f=\frac{g}{h} \in \mathcal{M}(\mathbb{K})$ have all its residues null, with $g \in \mathcal{A}(\mathbb{K})$ and $h \in \mathcal{A}^{0}(\mathbb{K})$ and $\psi(h)<+\infty$. Then for every $b \in \mathbb{K}, f-b$ has infinitely many zeros.

Remark: Consider a function $f$ of the form $\sum_{n=1}^{\infty} \frac{1}{\left(x-a_{n}\right)^{2}}$ with $\left|a_{n}\right|=n^{t}$. Clearly $f$ belongs to $\mathcal{M}(\mathbb{K})$, all residues are null, hence $f$ admits primitives. Next, primitives satisfy the hypothesis of Theorem F. Consequently, $f$ takes every value infinitely many times. Therefore, $f$ cannot be of the form $\frac{P}{h}$ with $P \in \mathbb{K}[x]$ and $h \in \mathcal{A}(\mathbb{K})$. 


\section{Type of growth}

Definition and notation: In complex analysis, the type of growth is defined for an entire function of order $t<+\infty$ as $\sigma(f)=\limsup _{r \rightarrow+\infty} \frac{\log \left(M_{f}(r)\right)}{r^{t}}$.

Of course the same notion may be defined for $f \in \mathcal{A}(\mathbb{K})$. Given $f \in \mathcal{A}^{0}(\mathbb{K})$ of order $t<+\infty$, we set $\sigma(f)=\limsup _{r \rightarrow+\infty} \frac{\log (|f|(r))}{r^{t}}$ and $\sigma(f)$ is called the type of growth of $f$.

Moreover, here we will also use the notation $\widetilde{\sigma}(f)=\liminf _{r \rightarrow+\infty} \frac{\log (|f| r))}{r^{t}}$.

Theorem 9: Let $f(x)=\sum_{n=0}^{\infty} a_{n} x^{n} \in \mathcal{A}^{0}(\mathbb{K})$ such that $\left.\rho(f) \in\right] 0,+\infty[$. Then

$$
\sigma(f) \rho(f) e=\limsup _{n \rightarrow+\infty}\left(n \sqrt[n]{\left|a_{n}\right| \rho(f)}\right)
$$

Proof: The proof is identical to this of the same statement in $\mathbb{C}[9]$, Proposition 11.5.

Notation: Let $f \in \mathcal{A}(\mathbb{K})$, let $\left(a_{n}\right)_{n \in \mathbb{N}}$ be the sequence of zeros of $f$ with $\left|a_{n}\right| \leq$ $\left|a_{n+1}\right|, n \in \mathbb{N}$ and for each $n \in \mathbb{N}$, let $w_{n}$ be the multiplicity order of $a_{n}$. For every $r>0$, let $k(r)$ be the integer such that $\left|a_{n}\right| \leq r \forall n \leq k(r)$ and $\left|a_{n}\right|>r \forall n>k(r)$. . We set $\psi(f, r)=\frac{q(f, r)}{r^{t}}$ and $\sigma(f, r)=\sum_{n=0}^{k(r)} \frac{w_{n}\left(\log (r)-\log \left(c_{n}\right)\right)}{r^{t}}$.

Theorem 10: Let $f \in \mathcal{A}^{0}(\mathbb{K})$. Then

$$
\rho(f)=\inf \{s \in] 0,+\infty\left[\mid \lim _{r \rightarrow+\infty} \frac{\log (|f|(r))}{r^{s}}=0\right\} .
$$

Proof: Indeed, let $M=\inf \{s \in] 0,+\infty\left[\mid \lim _{r \rightarrow+\infty} \frac{\log (|f|(r))}{r^{s}}=0\right\}$. First we will prove that $\rho(f) \leq M$ Let $s$ be such that $\lim _{r \rightarrow+\infty} \frac{\log (|f|(r))}{r^{s}}=0$. Let us fix $\epsilon>0$. For $r$ big enough, we have $\frac{\log (|f|(r))}{r^{s}} \leq \epsilon$, hence $\log (|f|(r)) \leq \epsilon r^{s}$, therefore $\log (\log (|f|(r))) \leq$ $\log \epsilon+s \log (r)$, hence $\frac{\log (\log (|f|(r)))}{\log (r)} \leq s+\frac{\epsilon}{\log (r)}$. This is true for every $\epsilon>0$, therefore $\limsup _{r \rightarrow+\infty} \frac{\log (\log (|f|(r)))}{\log (r)} \leq s$ i.e. $\rho(f) \leq s$ and hence, $\rho(f) \leq M$.

On the other hand, we notice that

$$
M=\sup \{s \in] 0,+\infty\left[\mid \limsup _{r \rightarrow+\infty} \frac{\log (|f|(r))}{r^{s}}>0\right\}
$$


Now, suppose that for some $s>0$, we have $\limsup _{r \rightarrow+\infty} \frac{\log (|f|(r))}{r^{s}}=b>0$. Let us fix $\epsilon \in] 0, b\left[\right.$. There exists a sequence $\left(r_{n}\right)_{n \in \mathbb{N}}$ such that, when $n$ is big enough, we have $b-\epsilon \leq \frac{\log \left(|f|\left(r_{n}\right)\right)}{\left(r_{n}\right)^{s}} \leq b+\epsilon$, hence

$s \log \left(r_{n}\right)+\log (b-\epsilon)<\log \left(\log \left(|f|\left(r_{n}\right)\right)\right)<s \log \left(r_{n}\right)+\log (b-\epsilon)$ therefore

$$
s+\frac{\log (b-\epsilon)}{\log \left(r_{n}\right)}<\frac{\log \left(\operatorname { l o g } \left(|f|\left(r_{n}\right)\right.\right.}{\log \left(r_{n}\right)}<s+\frac{\log (b+\epsilon)}{\log \left(r_{n}\right)}
$$

Consequently, $\lim _{n \rightarrow+\infty} \frac{\log \left(\log \left(|f|\left(r_{n}\right)\right)\right.}{\log \left(r_{n}\right)}=s$ and therefore $\rho(f) \geq s$, hence $\rho(f) \geq M$. Finally, $\rho(f)=M$.

Theorem 11: Let $f, g \in \mathcal{A}^{0}(\mathbb{K})$. Then $\sigma(f g) \leq \sigma(f)+\sigma(g)$. If $\rho(f) \geq \rho(g)$, then $\sigma(f) \leq \sigma(f g)$. If $\rho(f)=\rho(g)$, then $\max (\sigma(f), \sigma(g)) \leq \sigma(f g)$.

If $\rho(f)=\rho(g)$ and $\sigma(f)>\sigma(g)$ then $\rho(f+g)=\rho(f)$ and $\sigma(f+g)=\sigma(f)$. If $\rho(f+g)=\rho(f) \geq \rho(g)$ then $\sigma(f+g) \leq \max (\sigma(f), \sigma(g))$.

Proof: Let $s=\rho(f), t=\rho(g)$ and suppose $s \geq t$. When $r$ is big enough, we have $\max (\log (|f|(r)), \log (|g|(r)) \leq \log (|f . g|(r))=\log (|f|(r))+\log (|g|(r))$ and by Theorem 1, we have $\rho(f g)=s$. Therefore

$$
\begin{aligned}
\sigma(f g)= & \limsup _{r \rightarrow+\infty}\left(\frac{\log (|f \cdot g|(r))}{r^{s}}\right) \leq \limsup _{r \rightarrow+\infty}\left(\frac{\log (|f|(r))}{r^{s}}\right)+\limsup _{r \rightarrow+\infty}\left(\frac{\log (|g|(r))}{r^{s}}\right) \\
& \leq \limsup _{r \rightarrow+\infty}\left(\frac{\log (|f|(r))}{r^{s}}\right)+\limsup _{r \rightarrow+\infty}\left(\frac{\log (|g|(r))}{r^{t}}\right)=\sigma(f)+\sigma(g) .
\end{aligned}
$$

On the other hand,

$$
\sigma(f)=\limsup _{r \rightarrow+\infty} \frac{\log (|f|(r))}{r^{s}} \leq \limsup _{r \rightarrow+\infty} \frac{\log (|f g|(r))}{r^{s}} .
$$

But $\rho(f g)=s$, hence $\sigma(f) \leq \sigma(f g)$. Particularly, if $\rho(f)=\rho(g)$, then $\max (\sigma(f), \sigma(g)) \leq$ $\sigma(f g)$.

Now, suppose $s>t$. Then by Corollary 1.1, $\rho(f+g)=\rho(f)=s$. Consequently,

$$
\begin{array}{r}
\sigma(f+g)=\limsup _{r \rightarrow+\infty}\left(\frac{\log |f+g|(r)}{r^{s}}\right) \leq \limsup _{r \rightarrow+\infty}\left(\frac{\max (\log |f|(r), \log |g|(r))}{r^{s}}\right) \\
=\max \left(\limsup _{r \rightarrow+\infty}\left(\frac{\log |f|(r)}{r^{s}}\right), \limsup _{r \rightarrow+\infty}\left(\frac{\log |g|(r)}{r^{s}}\right)\right) \\
\leq \max \left(\limsup _{r \rightarrow+\infty}\left(\frac{\log |f|(r)}{r^{s}}\right), \limsup _{r \rightarrow+\infty}\left(\frac{\log |g|(r)}{r^{t}}\right)\right)=\max (\sigma(f), \sigma(g)) .
\end{array}
$$


Now, suppose $\rho(f)=\rho(g)=s$. Then

$$
\max \left(\limsup _{r \rightarrow+\infty}\left(\frac{\log (|f|(r))}{r^{s}}\right), \limsup _{r \rightarrow+\infty}\left(\frac{\log (|g|(r))}{r^{s}}\right)\right) \leq \limsup _{r \rightarrow+\infty}\left(\frac{\log (|f \cdot g|(r))}{r^{s}}\right)
$$

because the two both $|f|(r)$ and $|g|(r)$ tend to $+\infty$ with $r$. Consequently, $\sigma(f g) \geq$ $\max (\sigma(f), \sigma(g))$.

Now, suppose again that $\rho(f)=\rho(g)$ and suppose $\sigma(f)>\sigma(g)$. Let $s=\rho(f), b=$ $\sigma(f)$. Then $b>0$. Let $\left(r_{n}\right)_{n \in \mathbb{N}}$ be a sequence such that $\lim _{n \rightarrow+\infty} r_{n}=+\infty$ and $\lim _{n \rightarrow+\infty} \frac{\log \left(|f|\left(r_{n}\right)\right)}{\left(r_{n}\right)^{s}}=b$. Since $\sigma(g)<\sigma(f)$, we notice that when $n$ is big enough we have $|g|\left(r_{n}\right)<|f|\left(r_{n}\right)$. Consequently, when $n$ is big enough, we have $|f+g|\left(r_{n}\right)=|f|\left(r_{n}\right)$ and hence

$$
\lim _{n \rightarrow+\infty} \frac{\log \left(|f+g|\left(r_{n}\right)\right)}{\left(r_{n}\right)^{s}}=b
$$

By definition of $\sigma$ we have $\sigma(f+g) \geq \lim _{n \rightarrow+\infty} \frac{\log \left(|f+g|\left(r_{n}\right)\right)}{\left(r_{n}\right)^{\rho(f+g)}}$. By Theorem 1, we have $\rho(f+g) \leq s$, hence

$$
\begin{aligned}
& \sigma(f+g) \geq \lim _{n \rightarrow+\infty} \frac{\log \left(|f+g|\left(r_{n}\right)\right)}{\left(r_{n}\right)^{\rho(f+g)}} \geq \lim _{n \rightarrow+\infty} \frac{\log \left(|f+g|\left(r_{n}\right)\right)}{\left(r_{n}\right)^{s}} \\
& =\lim _{n \rightarrow+\infty} \frac{\log \left(|f|\left(r_{n}\right)\right)}{\left(r_{n}\right)^{s}}=\sigma(f)
\end{aligned}
$$

therefore by (1), $\sigma(f+g) \geq \sigma(f)$. Suppose that $\sigma(f+g)>\sigma(f)$. Putting $h=f+g$, we have $f=h-g$ with $\sigma(g)<\sigma(h)$, hence $\sigma(h-g) \geq \sigma(h)$ i.e. $\sigma(f)>\sigma(f+g)$, a contradiction. Consequently, $\sigma(f+g)=\sigma(f)$. Now we have, $\limsup _{r \rightarrow+\infty} \frac{\log (|f+g|(r))}{r^{s}}=b>0$. But then, $\limsup _{r \rightarrow+\infty} \frac{\log (|f+g|(r))}{r^{m}}=0 \forall m>s$. Therefore, by Theorem 10, $\rho(f+g)=\rho(f)$.

Finally, suppose now that $\rho(f+g)=\rho(f) \geq \rho(g)$. Let $s=\rho(f)$ and $t=\rho(g)$. Then,

$$
\begin{gathered}
\sigma(f+g)=\limsup _{r \rightarrow+\infty} \frac{\log (|f+g|(r))}{r^{s}} \leq \max \left(\limsup _{r \rightarrow+\infty} \frac{\log (|f|(r))}{r^{s}}, \limsup _{r \rightarrow+\infty} \frac{\log (|g|(r))}{r^{s}}\right) \\
=\max (\sigma(f), \sigma(g)) .
\end{gathered}
$$

Corollary 11.1: Let $f, g \in \mathcal{A}(\mathbb{K})$ be such that $\rho(f) \neq \rho(g)$. Then $\sigma(f+g) \leq$ $\max (\sigma(f), \sigma(g))$.

Proof: Indeed, suppose for instance $\rho(f)>\rho(g)$. Then $\rho(f+g)=\rho(f)$ hence, by the last assertion of Theorem 11, $\sigma(f+g) \leq \max (\sigma(f), \sigma(g))$. 
In the proof of Theorem 12, we will use the following trivial lemma:

Lemma $\mathbf{L}:$ Let $g, h$ be the real functions defined in $] 0,+\infty\left[\right.$ as $g(x)=\frac{e^{t x}-1}{x}$ and $h(x)=\frac{1-e^{-t x}}{x}$ with $t>0$. Then:

i) $\inf \{|g(x)| \mid x>0\}=t$.

ii) $\sup \{|h(x)| \mid x>0\}=t$.

Theorem 12: Let $f \in \mathcal{A}^{0}(\mathbb{K})$ be not identically zero. Then

$$
\rho(f) \sigma(f) \leq \psi(f) \leq \rho(f)(e \sigma(f)-\widetilde{\sigma}(f))
$$

Moreover, if $\psi(f)=\lim _{r \rightarrow+\infty} \frac{q(f, r)}{r^{\rho(f)}}$ or if $\sigma(f)=\lim _{r \rightarrow+\infty} \frac{\log (|f|(r))}{r^{\rho(f)}}$, then $\psi(f)=\rho(f) \sigma(f)$.

Proof: Without loss of generality we can assume that $f(0) \neq 0$. Let $t=\rho(f)$ and set $\ell=\log \left(\mid f(0 \mid)\right.$. Let $\left(a_{n}\right)_{n \in \mathbb{N}}$ be the sequence of zeros of $f$ with $\left|a_{n}\right| \leq\left|a_{n+1}\right|, n \in \mathbb{N}$ and for each $n \in \mathbb{N}$, let $w_{n}$ be the multiplicity order of $a_{n}$. For every $r>0$, let $k(r)$ be the integer such that $\left|a_{n}\right| \leq r \forall n \leq k(r)$ and $\left|a_{n}\right|>r \forall n>k(r)$. Then by Theorem A, we have $\log (|f|(r))=\ell+\sum_{n=0}^{k(r)} w_{n}\left(\log (r)-\log \left(\left|a_{n}\right|\right)\right)$ hence

$$
\sigma(f)=\limsup _{r \rightarrow+\infty}\left(\frac{\ell+\sum_{n=0}^{k(r)} w_{n}\left(\log (r)-\log \left(\left|a_{n}\right|\right)\right)}{r^{t}}\right) .
$$

Given $r>0$, set $c_{n}=\left|a_{n}\right|$ and let us keep the notations above. Then

$$
\sigma(f)=\limsup _{r \rightarrow+\infty} \sigma(f, r), \quad \psi(f)=\limsup _{r \rightarrow+\infty} \psi(f, r) .
$$

We will first show the inequality $\rho(f) \sigma(f) \leq \psi(f)$. By the definition of $\sigma(f, r)$ we can derive

$$
\begin{gathered}
\sigma(f, r) \leq \sum_{n=0}^{k\left(r e^{-\alpha}\right)} \frac{w_{n}\left(\log (r)-\log \left(r e^{-\alpha}\right)\right)}{r^{t}} \\
+\sum_{n=0}^{k\left(r e^{-\alpha}\right)} \frac{w_{n}\left(\log \left(r e^{-\alpha}\right)-\log \left(c_{n}\right)\right)}{r^{t}}+\alpha \sum_{k\left(r e^{-\alpha}\right)<n \leq k(r)} \frac{w_{n}}{r^{t}}
\end{gathered}
$$

hence

$$
\begin{gathered}
\sigma(f, r) \leq \alpha \sum_{n=0}^{k\left(r e^{-\alpha}\right)} \frac{w_{n}}{r^{t}}+\sum_{n=0}^{k\left(r e^{-\alpha}\right)} \frac{w_{n}\left(\log \left(r e^{-\alpha}\right)-\log \left(c_{n}\right)\right)}{r^{t}} \\
+\alpha \sum_{k\left(r e^{-\alpha}\right)<n \leq k(r)} \frac{w_{n}}{r^{t}}
\end{gathered}
$$


therefore

$$
\begin{gathered}
\sigma(f, r) \leq \alpha \sum_{n=0}^{k\left(r e^{-\alpha}\right)} \frac{w_{n}}{r^{t}}+\sum_{n=0}^{k\left(r e^{-\alpha}\right)} \frac{w_{n}\left(\log \left(r e^{-\alpha}\right)-\log \left(c_{n}\right)\right)}{r^{t}} \\
+\alpha \sum_{k\left(r e^{-\alpha}\right)<n \leq k(r)} \frac{w_{n}}{r^{t}}
\end{gathered}
$$

hence

$$
\begin{aligned}
& \sigma(f, r) \leq \alpha \sum_{n=0}^{k\left(r e^{-\alpha}\right)} \frac{w_{n}}{r^{t}}+e^{-t \alpha} \sum_{n=0}^{k\left(r e^{-\alpha}\right)} \frac{w_{n}\left(\log \left(r e^{-\alpha}\right)-\log \left(c_{n}\right)\right)}{\left(r e^{-\alpha}\right)^{t}} \\
&+\alpha \sum_{0 \leq n \leq k(r)} \frac{w_{n}}{r^{t}}-\alpha \sum_{0 \leq n \leq k\left(r e^{-\alpha}\right)} \frac{w_{n}}{r^{t}},
\end{aligned}
$$

hence

$$
\sigma(f, r) \leq e^{-t \alpha} \sum_{n=0}^{k\left(r e^{-\alpha}\right)} \frac{w_{n}\left(\log \left(r e^{-\alpha}\right)-\log \left(c_{n}\right)\right)}{\left(r e^{-\alpha}\right)^{t}}+\alpha \sum_{0 \leq n \leq k(r)} \frac{w_{n}}{r^{t}}
$$

Thus we have

$$
\sigma(f, r) \leq e^{-t \alpha} \sigma\left(f, r e^{-\alpha}\right)+\alpha \psi(f, r) .
$$

We check that we can pass to superior limits on both sides, so we obtain $\sigma(f) \leq e^{-t \alpha} \sigma(f)+$ $\alpha \psi(f)$ therefore $\sigma(f) \frac{\left(1-e^{-t \alpha}\right)}{\alpha} \leq \psi(f)$. That holds for every $\alpha>0$, hence by Lemma $\mathrm{L}$ ii), we can derive

$$
\psi(f) \geq \rho(f) \sigma(f)
$$

We will now show the inequality

$$
\psi(f) \leq \rho(f)(e \sigma(f)-\widetilde{\sigma}(f))
$$

Let us fix $\alpha>0$. We can write

$$
\begin{gathered}
\sigma(f, r)=\sum_{n=0}^{k\left(r e^{-\alpha}\right)} \frac{w_{n}\left(\log (r)-\log \left(r e^{-\alpha}\right)\right)}{r^{t}} \\
+\sum_{j=0}^{k\left(r e^{-\alpha}\right)} \frac{w_{j}\left(\log \left(r e^{-\alpha}\right)-\log \left(c_{n}\right)\right)}{r^{t}}+\sum_{k\left(r e^{-\alpha}\right)<j \leq k(r)} \frac{w_{j}\left(\log (r)-\log \left(c_{j}\right)\right)}{r^{t}}
\end{gathered}
$$


hence

$$
\sigma(f, r) \geq \alpha \sum_{n=0}^{k\left(r e^{-\alpha}\right)} \frac{w_{n}}{r^{t}}+\sum_{j=0}^{k\left(r e^{-\alpha}\right)} \frac{w_{j}\left(\log \left(r e^{-\alpha}\right)-\log \left(c_{n}\right)\right)}{r^{t}}
$$

hence

$$
\sigma(f, r) \geq \alpha e^{-t \alpha} \sum_{n=0}^{k\left(r e^{-\alpha}\right)} \frac{w_{n}}{\left(r e^{-t \alpha}\right)}+e^{-t \alpha} \sum_{j=0}^{k\left(r e^{-\alpha}\right)} \frac{w_{n}\left(\log \left(r e^{-\alpha}\right)-\log \left(c_{n}\right)\right)}{\left(r e^{-\alpha}\right)^{t}}
$$

and hence

$$
\sigma(f, r) \geq \alpha \psi\left(f, r e^{-\alpha}\right)+e^{-t \alpha} \sigma\left(f, r e^{-\alpha}\right) .
$$

Therefore, we can derive

$$
\left.\alpha e^{-t \alpha} \psi(f) \leq \limsup _{r \rightarrow+\infty}\left(\sigma(f, r)-e^{-t \alpha} \sigma\left(f, r e^{-\alpha}\right)\right)\right)
$$

and therefore

$$
\left.\alpha e^{-t \alpha} \psi(f) \leq \sigma(f)-e^{-t \alpha} \widetilde{\sigma}(f)\right) .
$$

That holds for every $\alpha>0$ and hence, when $t \alpha=1$, we obtain $\psi(f) \leq \rho(f)(e \sigma(f)-\widetilde{\sigma}(f))$ which is the left hand inequality of the general conclusion.

Now, suppose that $\sigma(f)=\lim _{r \rightarrow+\infty} \frac{\log (|f|(r))}{r^{t}}$. Then we have $\limsup _{r \rightarrow+\infty} \psi(f, r) \leq \sigma(f)\left(\frac{e^{t \alpha}-1}{\alpha}\right)$ and hence $\psi(f) \leq \sigma(f)\left(\frac{e^{t \alpha}-1}{\alpha}\right)$. That holds for every $\alpha>0$ and then, by Lemma L i) we obtain $\psi(f) \leq t \sigma(f)$, i.e. $\psi(f) \leq \rho(f) \sigma(f)$, hence by (2) we have, $\psi(f)=\rho(f) \sigma(f)$.

Now, suppose that

$$
\psi(f)=\lim _{r \rightarrow+\infty} \sum_{n=0}^{k(r)} \frac{w_{n}}{r^{t}}=\lim _{r \rightarrow+\infty} \psi(f, r)
$$

We can obviously find a sequence $\left(r_{n}\right)_{n \in \mathbb{N}}$ in $] 0,+\infty[$ of limit $+\infty$ such that $\sigma(f)=$ $\lim _{n \rightarrow+\infty} \sigma\left(f, r_{n} e^{-\alpha}\right)$. Then, by (1) we have

$$
\sigma\left(f, r_{n}\right) \geq \alpha e^{-t \alpha} \psi\left(f, \frac{r_{n}}{e^{\alpha}}\right)+e^{-t \alpha} \sigma\left(f, \frac{r_{n}}{e^{\alpha}}\right)
$$

hence

$$
\limsup _{n \rightarrow+\infty} \sigma\left(f, r_{n}\right) \geq \alpha e^{-t \alpha} \psi(f)+e^{-t \alpha} \sigma(f)
$$

and hence

$$
\sigma(f) \geq \alpha e^{-t \alpha} \psi(f)+e^{-t \alpha} \sigma(f)
$$


therefore, $\psi(f) \leq\left(\frac{e^{t \alpha}-1}{\alpha}\right) \sigma(f)$. Finally, by Lemma L i) we have, $\psi(f) \leq \rho(f) \sigma(f)$ and hence by $(2), \psi(f)=\rho(f) \sigma(f)$.

Remarks: 1) When neither $\sigma$ nor $\psi$ are obtained as veritable limits when $r$ tends to $+\infty$, the method does not let us prove that $\psi=\rho \sigma$, the natural conjecture.

2) Concerning the upper bound $\psi(f) \leq \rho(f)(e \sigma(f)-\widetilde{\sigma}(f))$ it is possible to improve this a bit by defining the number $u_{0}>0$ such that $e^{u_{0}}\left(u_{0}-1\right)=-\frac{\tilde{\sigma}(f)}{\sigma(f)}$ and then we have

$$
\psi(f) \leq \frac{\rho(f)\left(e^{u_{0}} \sigma(f)-\tilde{\sigma}(f)\right)}{u_{0}}
$$

Corollary 12.1: Let $f \in \mathcal{A}(\mathbb{K})$ be not identically zero and have finite growth order. Then $\sigma(f)$ is finite if and only if so is $\psi(f)$.

By Theorems 9 and 12 we can also notice this corollary:

Corollary 12.2: Let $f(x)=\sum_{n=0}^{\infty} a_{n} x^{n} \in \mathcal{A}^{0}(\mathrm{IK})$ be not identically zero. Then

$$
\limsup _{n \rightarrow+\infty}\left(n \sqrt[n]{\left|a_{n}\right|^{\rho(f)}}\right) \leq e \psi(f) \leq e \limsup _{n \rightarrow+\infty}\left(n \sqrt[n]{\left|a_{n}\right|^{\rho(f)}}\right)
$$

Moreover, if $\psi(f)=\lim _{r \rightarrow+\infty} \frac{q(f, r)}{r^{\rho(f)}}$ or if $\sigma(f)=\lim _{r \rightarrow+\infty} \frac{\log (|f|(r))}{r^{\rho(f)}}$, then

$$
e \psi(f)=\limsup _{n \rightarrow+\infty}\left(n \sqrt[n]{\left|a_{n}\right|^{\rho(f)}}\right)
$$

Theorem 12 suggests the following conjecture:

Conjecture 1: Let $f \in \mathcal{A}^{0}(\mathbb{K})$ be such that either $\sigma(f)<+\infty$ or $\psi(f)<+\infty$. Then $\psi(f)=\rho(f) \sigma(f)$.

Now, by Theorem 8, we can also state Corollary 12.3:

Corollary 12.3: Let $f=\frac{g}{h} \in \mathcal{M}(\mathbb{K})$, with $g, h \in \mathcal{A}(\mathbb{K})$ not identically zero and be such that $h$ has finite order of growth and and finite type of growth. Then $f^{\prime}$ takes every value $b \in \mathbb{K}$ infinitely many times.

\section{Order and type of the derivative}

Theorem 13: Let $f \in \mathcal{A}(\mathbb{K})$ be not identically zero. Then $\rho(f)=\rho\left(f^{\prime}\right)$. 
Proof: By Theorem 6 we have $\rho\left(f^{\prime}\right)=\limsup _{n \rightarrow+\infty}\left(\frac{n \log (n)}{-\log \left(\left|(n+1) a_{n+1}\right|\right)}\right)$. But since $\frac{1}{n} \leq$ $|n| \leq 1$, we have

$$
\begin{gathered}
\limsup _{n \rightarrow+\infty}\left(\frac{n \log (n)}{-\log \left(\left|(n+1) a_{n+1}\right|\right.}\right)=\limsup _{n \rightarrow+\infty}\left(\frac{n \log (n)}{-\log \left(\left|a_{n+1}\right|\right)}\right) \\
=\limsup _{n \rightarrow+\infty}\left(\frac{(n+1) \log (n+1)}{-\log \left(\left|a_{n+1}\right|\right)}\right)=\rho(f) .
\end{gathered}
$$

Corollary 13.1: The derivation on $\mathcal{A}(\mathbb{K})$ restricted to the algebra $\mathcal{A}(\mathbb{K}, t)$ (resp. to $\mathcal{A}^{0}(\mathbb{K})$ ) provides that algebra with a derivation.

In complex analysis, it is known that if an entire function $f$ has order $t<+\infty$, then $f$ and $f^{\prime}$ have same type. We will check that it is the same here.

Theorem 14: Let $f \in \mathcal{A}(\mathbb{K})$ be not identically zero, of order $t \in] 0,+\infty[$. Then $\sigma(f)=$ $\sigma\left(f^{\prime}\right)$.

Proof: $\quad$ By Theorem 8 we have, $e \rho\left(f^{\prime}\right) \sigma\left(f^{\prime}\right)$

$$
\begin{gathered}
=\limsup _{n \rightarrow+\infty}\left(n\left(|n+1|\left|a_{n+1}\right|\right)^{\frac{t}{n}}\right)=\limsup _{n \rightarrow+\infty}\left(\left((n+1)\left(|n+1|\left|a_{n+1}\right|\right)^{\frac{t}{n}}\right)^{\frac{n}{n+1}}\left(\frac{n}{n+1}\right)\right) \\
=\limsup _{n \rightarrow+\infty}\left((n+1)\left(|n+1|\left|a_{n+1}\right|\right)^{\frac{t}{n+1}}\right)=e \rho(f) \sigma(f) .
\end{gathered}
$$

But since $\rho(f)=\rho\left(f^{\prime}\right)$ and since $\rho(f) \neq 0$, we can see that $\sigma\left(f^{\prime}\right)=\sigma(f)$.

By Theorems 12, 13, 14 we can now derive

Corollary 14.1: Let $f \in \mathcal{A}^{0}(\mathbb{K})$ be not identically zero. Then $\rho(f) \sigma(f) \leq \psi\left(f^{\prime}\right) \leq e \rho(f) \sigma(f)$ and $\left|\psi\left(f^{\prime}\right)-\psi(f)\right|_{\infty} \leq(e-1) \rho(f) \sigma(f)$.

Corollary 14.2: Let $f \in \mathcal{A}^{0}(\mathbb{K})$ be not identically zero. Then $\psi\left(f^{\prime}\right)$ is finite if and only if so is $\psi(f)$.

Corollary 14.3: Let $f \in \mathcal{A}^{0}(\mathbb{K})$ be not identically zero. Then in each one of the following hypotheses, we have $\psi\left(f^{\prime}\right)=\psi(f)=\rho(f) \sigma(f)$ :

i) $\psi(f)=\lim _{r \rightarrow+\infty} \psi(f, r)$ and $\psi\left(f^{\prime}\right)=\lim _{r \rightarrow+\infty} \psi\left(f^{\prime}, r\right)$,

ii) $\sigma(f)=\lim _{r \rightarrow+\infty} \sigma(f, r)$ and $\sigma\left(f^{\prime}\right)=\lim _{r \rightarrow+\infty} \sigma\left(f^{\prime}, r\right)$,

iii) $\psi(f)=\lim _{r \rightarrow+\infty} \psi(f, r)$ and $\sigma\left(f^{\prime}\right)=\lim _{r \rightarrow+\infty} \sigma\left(f^{\prime}, r\right)$,

iv) $\sigma(f)=\lim _{r \rightarrow+\infty} \sigma(f, r)$ and $\psi\left(f^{\prime}\right)=\lim _{r \rightarrow+\infty} \psi\left(f^{\prime}, r\right)$.

By Corollary 12.3, we have the following corollary: 
Corollary 14.4: Let $f=\frac{g}{h} \in \mathcal{M}(\mathbb{K})$ be not identically zero, with $g, h \in \mathcal{A}(\mathbb{K})$ and suppose that $f$ has all residues null and that $h$ has finite order of growth and finite type of growth. Then $f$ takes every value $b \in \mathbb{K}$ infinitely many times.

Conjecture 1 suggests and implies the following Conjecture 2:

Conjecture 2: $\quad \psi(f)=\psi\left(f^{\prime}\right) \forall f \in \mathcal{A}^{0}(\mathbb{K})$.

Theorem 15: Let $f, g \in \mathcal{A}(\mathbb{K})$ be transcendental and of same order $t \in[0,+\infty[$. Then for every $\epsilon>0$, we have

$$
\limsup _{r \rightarrow+\infty}\left(\frac{r^{\epsilon} q(g, r)}{q(f, r)}\right)=+\infty
$$

Proof: Suppose first $t=0$. The proof then is almost trivial. Indeed, for all $\epsilon>0$, we have $\lim _{r \rightarrow+\infty} \frac{q(f, r)}{r^{\epsilon}}=0$ hence $\lim _{r \rightarrow+\infty} \frac{r^{\epsilon}}{q(f, r)}=+\infty$, therefore $\lim _{r \rightarrow+\infty} \frac{r^{\epsilon} q(g, r)}{q(f, r)}=+\infty$.

Now suppose $t>0$. By Theorem 4, we have $\limsup _{r \rightarrow+\infty} \frac{q(f, r)}{r^{t}}$ is a finite number $\ell$ and hence there exists $\lambda>0$ such that

$$
q(f, r) \leq \lambda r^{t} \forall r>1
$$

Now, let us fix $s \in] 0, t[$. By hypothesis, $\rho(g)=\rho(f)$ and hence by Theorem 4 , we have $\limsup _{r \rightarrow+\infty} \frac{q(g, r)}{r^{s}}=+\infty$ so, there exists an increasing sequence $\left(r_{n}\right)_{n \in \mathbb{N}}$ of $\mathbb{R}_{+}$such that $\lim _{n \rightarrow+\infty} r_{n}=+\infty$ and $\frac{q\left(g, r_{n}\right)}{\left(r_{n}\right)^{s}} \geq n$. Therefore, by (1), we have

$$
\frac{\lambda\left(r_{n}\right)^{t} q\left(g, r_{n}\right)}{\left(r_{n}\right)^{s} q\left(f, r_{n}\right)}>\frac{q\left(g, r_{n}\right)}{\left(r_{n}\right)^{s}}>n
$$

and hence

$$
\lambda \lim _{n \rightarrow+\infty}\left(\frac{\left(r_{n}\right)^{t-s} q\left(g, r_{n}\right)}{q\left(f, r_{n}\right)}\right)=+\infty
$$

Consequently,

$$
\limsup _{r \rightarrow+\infty}\left(\frac{(r)^{t-s} q(g, r)}{q(f, r)}\right)=+\infty
$$

Now, since that holds for all $s \in] 0, t[$, the statement derived from (2).

Remark: Comparing the number of zeros of $f^{\prime}$ to this of $f$ inside a disk is very uneasy. Now, we can give some precisions. By Theorem 15 we can derive Corollary 15.1. 
Corollary 15.1: Let $f \in \mathcal{A}^{0}(\mathbb{I K})$ be not affine. Then for every $\epsilon>0$, we have

$$
\limsup _{r \rightarrow+\infty}\left(\frac{r^{\epsilon} q\left(f^{\prime}, r\right)}{q(f, r)}\right)=+\infty
$$

and

$$
\limsup _{r \rightarrow+\infty}\left(\frac{r^{\epsilon} q(f, r)}{q\left(f^{\prime}, r\right)}\right)=+\infty
$$

We can now give a partial solution to a problem that arose when studying the zeros of derivatives of meromorphic functions: given $f \in \mathcal{A}(\mathbb{K})$, is it possible that $f^{\prime}$ divides $f$ in the algebra $\mathcal{A}(\mathbb{K})$ ?

Theorem 16: Let $f \in \mathcal{A}(\mathbb{K}) \backslash \mathbb{K}[x]$. Suppose that for some number $s>0$ we have $\limsup |q(f, r)| r^{s}>0$ (where $|q(f, r)|$ is the ultrametric absolute value of $q(f, r)$ defined on $\mathrm{IK})$. Then $f^{\prime}$ has infinitely many zeros that are not zeros of $f$.

Proof: Suppose that $f^{\prime}$ only has finitely many zeros that are not zeros of $f$. Then there exist $h \in \mathcal{A}(\mathbb{K})$ and $P \in \mathbb{K}[x]$ such that $P f=f^{\prime} h$. Without loss of generality, we can assume that $P$ is monic. Every zero of $f$ of order $u \geq 2$ is a zero of $f^{\prime}$ of order $u-1$ and hence is a zero of $h$. And every zero of $f$ of order 1 is zero of $h$ of order 1 too. Consequently, $h$ is not a polynomial.

Set $f(x)=\sum_{n=0}^{\infty} a_{n} x^{n}, f^{\prime}(x)=\sum_{n=0}^{\infty} c_{n} x^{n} h(x)=\sum_{n=0}^{\infty} b_{n} x^{n}$ and let $s=\operatorname{deg}(P)$. Then $c_{n}=(n+1) a_{n+1} \forall n \in \mathbb{N}$. On the other hand, by Theorem A, given any $r>0$ we have $|f|(r)=\left|a_{q(f, r)}\right| r^{q(f, r)},\left|f^{\prime}\right|(r)=\left|c_{q\left(f^{\prime}, r\right)}\right| r^{q\left(f^{\prime}, r\right)}=\left|\left(q\left(f^{\prime}, r\right)+1\right) a_{\left.q\left(f^{\prime}, r\right)+1\right)}\right| r^{q\left(f^{\prime}, r\right)}$ and $|h|(r)=\left|b_{q(h, r)}\right| r^{q(h, r)}$. Since $h$ has infinitely many zeros, there exists $r_{0}>0$ such that $q(h, r) \geq s+2 \forall r \geq r_{0}$, assuming that all zeros of $P$ belong to $d(0, s)$. Then since the norm $|\cdot|(r)$ is multiplicative, we have $s+q(f, r)=q\left(f^{\prime}, r\right)+q(h, r)$, hence

$$
q\left(f^{\prime}, r\right)<q(f, r)-1 \forall r \geq r_{0} .
$$

Then, by (1) we have $\left|c_{n}\right| r^{n}<c_{q\left(f^{\prime}, r\right)} r^{q\left(f^{\prime}, r\right)} \forall n>q\left(f^{\prime}, r\right), \forall r \geq r_{0}$ and particularly, $\left|c_{q(f, r)-1}\right| r^{q(f, r)-1}<\left|f^{\prime}\right|(r)=\left|c_{q\left(f^{\prime}, r\right)}\right| r^{q\left(f^{\prime}, r\right)}$ i.e.

$$
\left|(q(f, r)) a_{q(f, r)}\right| r^{(q(f, r)-1)}<\left|f^{\prime}\right|(r)=\left|\left(q\left(f^{\prime}, r\right)+1\right) a_{q\left(f^{\prime}, r\right)+1}\right| r^{q\left(f^{\prime}, r\right)}
$$

On the other hand, since $P f=f^{\prime} h$, we have $|P|(r)|f|(r)=\left|f^{\prime}\right|(r)|h|(r)$, hence since $P$ is monic,

$$
r^{s}\left|a_{q(f, r)}\right| r^{q(f, r)}=\left|\left(q\left(f^{\prime}, r\right)+1\right) a_{\left(q\left(f^{\prime}, r\right)+1\right)}\right| r^{q\left(f^{\prime}, r\right)}\left|b_{q(h, r)}\right| r^{q(h, r)} \forall r>r_{0} .
$$

By (2) we can derive

$$
r^{s-1}\left|q(f, r) a_{q(f, r)}\right| r^{q(f, r)}<r^{s}\left|\left(q\left(f^{\prime}, r\right)+1\right) a_{q\left(f^{\prime}, r\right)+1}\right| r^{q\left(f^{\prime}, r\right)}
$$


and by (3) we have

$$
\left(\frac{|q(f, r)|}{r}\right)\left|\left(q\left(f^{\prime}, r\right)+1\right) a_{q\left(f^{\prime}, r\right)+1}\right| r^{q\left(f^{\prime}, r\right)}\left|b_{q(h, r)}\right| r^{q(h, r)}<r^{s}\left|\left(q\left(f^{\prime}, r\right)+1\right) a_{q\left(f^{\prime}, r\right)+1}\right| r^{q\left(f^{\prime}, r\right)}
$$

therefore we obtain $\left|b_{q(h, r)}\right| r^{q(h, r)-1}|q(f, r)|<r^{s}$. Consequently,

$$
|h|(r)<\frac{r^{s+1}}{|q(f, r)|}
$$

Since $h$ is transcendental, we have $\lim _{r \rightarrow+\infty} \frac{|h|(r)}{r^{m}}=+\infty \forall m>0$. Now, suppose that for some integer $m$ we have $\limsup |q(f, r)| r^{m}>0$, hence there exists a constant $c$ and an increasing sequence $\left(r_{n}\right)_{n \in \mathbb{N}^{*}}$ such that $r_{1}>r_{0}, \lim _{n \rightarrow+\infty} r_{n}=+\infty$ and $\left|q\left(f, r_{n}\right)\right|\left(r_{n}\right)^{m}>$ $c \forall n$. Then $|h|\left(r_{n}\right)<c\left(r_{n}\right)^{s+1+m} \forall n$, a contradiction to (4). This finishes proving that $P$ and $h$ do not not exist.

Remark: It is possible to deduce the proof of Theorem 16 by using Lemma 1.4 in [3].

Corollary 16.1 is a very partial answer to the $p$-adic Hayman conjecture when $n=1$, which is not solved yet.

Corollary 16.1 Let $f \in \mathcal{M}(\mathbb{K})$ be such that

$$
\limsup _{r \rightarrow+\infty}\left|q\left(\frac{1}{f}, r\right)\right| r^{s}>0
$$

for some $s>0$. Then $f f^{\prime}$ has at least one zero.

Proof: Indeed, suppose that $f f^{\prime}$ has no zero. Then $f$ is of the form $\frac{1}{h}$ with $h \in \mathcal{A}(\mathbb{K})$ and $f^{\prime}=-\frac{h^{\prime}}{h^{2}}$ has no zero, hence every zero of $h^{\prime}$ is a zero of $h$, a contradiction to Theorem 16 since $\lim \sup _{r \rightarrow+\infty}|q(h, r)| r^{s}>0$.

Remarks: Concerning complex entire functions, we check that the exponential is of order 1 but is divided by its derivative in the algebra of complex entire functions.

It is also possible to derive Corollary 16.2 from Theorem 1 of [1]. Indeed, let $g=\frac{1}{f}$. By Theorem 4, $\limsup _{r \rightarrow+\infty} \frac{q(f, r)}{r^{t}}$ is a finite number. Consequently, there exists $c>0$ such that $q(f, r) \leq c r^{t} \forall r>1$ and therefore the number of poles of $g$ in $d(0, r)$ is upper bounded by $c r^{t}$ whenever $r>1$. Consequently, we can apply Theorem 8 and hence the meromorphic function $g^{\prime}$ has infinitely many zeros. Now, suppose that $f^{\prime}$ divides $f$ in $\mathcal{A}(\mathbb{I K})$. Then every zero of $f^{\prime}$ is a zero of $f$ with an order superior, hence $\frac{f^{\prime}}{f^{2}}$ has no zero, a contradiction. 
If the residue characteristic of $\mathbb{K}$ is $p \neq 0$, we can easily construct an example of entire function $f$ of infinite order such that $f^{\prime}$ does not divide $f$ in $\mathcal{A}(\mathbb{K})$. Let $f(x)=$ $\prod_{n=0}^{\infty}\left(1-\frac{x}{\alpha_{n}}\right)^{p^{n}}$ with $\left|\alpha_{n}\right|=n+1$. We check that $q(f, n+1)=\sum_{k=0}^{n} p^{k}$ is prime to $p$ for every $n \in \mathbb{N}$. Consequently, Theorem 16 shows that $f$ is not divided by $f^{\prime}$ in $\mathcal{A}(\mathbb{K})$. On the other hand, fixing $t>0$, we have

$$
\frac{q(f, n+1)}{(n+1)^{t}} \geq \frac{p^{n}}{(n+1)^{t}}
$$

hence

$$
\limsup _{r \rightarrow+\infty} \frac{q(f, r)}{r^{t}}=+\infty \forall t>0
$$

therefore, $f$ is not of finite order.

Theorem 16 suggests the following conjecture:

Conjecture 3 Given $f \in \mathcal{A}(\mathbb{K})$, other than $(x-a)^{m}, a \in \mathbb{K}, m \in \mathbb{N}$, there exists no $h \in \mathcal{A}(\mathbb{K})$ such that $f=f^{\prime} h$.

\section{References}

[1] J.P. Bezivin, K. Boussaf, A. Escassut, Zeros of the derivative of a p-adic meromorphic function, Bulletin des Sciences Mathématiques 136, n. 8, p.839-847 (2012).

[2] J.P. Bezivin, K. Boussaf, A. Escassut, Some new and old results on zeros of the derivative of a p-adic meromorphic function, Contemporary Mathematics, Amer. Math. Soc. vol. 596, p. 23-30 (2013).

[3] K. Boussaf, A. Escassut and J. Ojeda, Primitives of p-adic meromorphic functions, Contemporary mathematics vol 551, p.51-56 (2011)

[4] K. Boussaf, A. Boutabaa, A. Escassut, Growth of p-adic entire functions and applications Houston Journal of Mathematics (2014).

[5] A. Escassut, Analytic Elements in p-adic Analysis. World Scientific Publishing Co. Pte. Ltd. Singapore, (1995).

[6] A. Escassut, p-adic Value Distribution. Some Topics on Value Distribution and Differentability in Complex and P-adic Analysis, p. 42- 138. Mathematics Monograph, Series 11. Science Press.(Beijing 2008).

[7] A. Escassut, Value Distribution in p-adic Analysis. World Scientific Publishing Co. Pte. Ltd. Singapore, (2015).

[8] P.C. Hu and C.C. Yang, Meromorphic Functions over non-Archimedean Fields, Kluwer Academic Publishers, (2000).

[9] L. A. Rubel, Entire and meromorphic functions Springer-Verlag, New York, (1996). 
Kamal Boussaf, Abdelbaki Boutabaa, Alain Escassut, Laboratoire de Mathématiques, CNRS UMR 6620

Unversité Blaise Pascal

24 Avenue des Landais

63178 AUBIERE CEDEX

FRANCE

kamal.boussaf@math.univ-bpclermont.fr

abdelbaki.boutabaa@math.univ-bpclermont.fr

alain.escassut@math.univ-bpclermont.fr 